\title{
Epidemiological characteristics, clinical outcomes and management patterns of metastatic breast cancer patients in routine clinical care settings of Greece: Results from the EMERGE multicenter retrospective chart review study
}

Athanasios Kotsakis ${ }^{1}$, Alexandros Ardavanis ${ }^{2}$, Georgios Koumakis ${ }^{3}$, Epameinondas Samantas ${ }^{4}$, Amanta Psyrri ${ }^{5}$ and Christos Papadimitriou ${ }^{6^{*}}$ (D)

\begin{abstract}
Background: The "EMERGE" study, aimed to capture real-life management patterns and outcomes in metastatic breast cancer (MBC) in Greece, also accounting for hormone (HR) and human epidermal growth factor receptor 2 (HER2) status.

Methods: "EMERGE" was a multicenter, retrospective cohort study of adult MBC patients diagnosed between 01Janaury-2010 and 30-June-2012, either de novo or having progressed from a non-metastatic state. Patient data, including treatment patterns and outcomes, were mainly abstracted through medical chart review.

Results: 386 patients were enrolled by 16 hospital-based oncologists between 12-March-2013 and 31-March-2015. The median look-back period was 29.1 months. At MBC diagnosis, $56.1 \%$ of the patients were $\mathrm{HR}^{+} / \mathrm{HER}^{-}, 16.6 \%$ $\mathrm{HR}^{+} / \mathrm{HER2}^{+}, 14.5 \% \mathrm{HR}^{-} / \mathrm{HER2}^{-}$, and $12.8 \% \mathrm{HR}^{-} / \mathrm{HER2}^{+}$. In the first line setting, chemotherapy, targeted therapy and endocrine therapy were received by $76.7,52.4$, and $28.3 \%$ of the overall population, and by $66.5 / 36.2 / 42.0 \%, 80.4 / 80$. 4/28.6\%, 88.4/90.7/0.0, and 95.6\%/56.5/6.5\% of the $\mathrm{HR}^{+} / \mathrm{HER2}^{-}, \mathrm{HR}^{+} / \mathrm{HER2}^{+}, \mathrm{HR}^{-} / \mathrm{HER2}^{+}$, $\mathrm{HR}^{-} / \mathrm{HER2}^{-}$subpopulations, respectively. In the overall population, the disease progression incidence rate was 0.57 [95\% confidence interval (Cl): 0.48-0.67] per person-year; median progression-free survival (PFS) was 22.4 (95\% Cl: 20.4-24.7) and overall survival (OS) was 45.0 (95\% Cl: 40.9-55.0) months. Median PFS was 24.6 (95\% Cl: 21.3-27.9) in HR+/HER2-, 19.7 (95\% Cl: 12. 9-25.9) in $\mathrm{HR}^{+} / \mathrm{HER2}^{+}, 23.0$ (95\% Cl: 16.6-29.7) in $\mathrm{HR}^{-} / \mathrm{HER2}^{+}$and 18.3 (95\% Cl: 10.0-24.7) months in $\mathrm{HR}^{-} / \mathrm{HER}^{-}$ subpopulations. A multivariable Cox proportional hazards model, adjusted among other factors for age and duration of diagnosis, HR and HER2 status, demonstrated that in the overall population PFS was better among those receiving first line endocrine therapy (hazard ratio: $0.70 ; 95 \% \mathrm{Cl}: 0.51-0.95 ; p=0.024$ ).
\end{abstract}

Conclusions: "EMERGE" demonstrates differences between HR/HER2 subtypes in clinical outcomes and divergence from evidence-based guideline recommendations for MBC management, especially as it pertains to the $\mathrm{HR}^{+} / \mathrm{HER}^{-}$ patients in which chemotherapy was favored over endocrine therapy in the first line setting.

(Continued on next page)

\footnotetext{
* Correspondence: chr_papadim@yahoo.gr

${ }^{6}$ Oncology Unit, 2nd Department of Surgery, Aretaieion Hospital, 76 Vas.

Sofias Av, 11528 Athens, Greece

Full list of author information is available at the end of the article
}

(c) The Author(s). 2019 Open Access This article is distributed under the terms of the Creative Commons Attribution 4.0 International License (http://creativecommons.org/licenses/by/4.0/), which permits unrestricted use, distribution, and reproduction in any medium, provided you give appropriate credit to the original author(s) and the source, provide a link to the Creative Commons license, and indicate if changes were made. The Creative Commons Public Domain Dedication waiver (http://creativecommons.org/publicdomain/zero/1.0/) applies to the data made available in this article, unless otherwise stated. 
(Continued from previous page)

Study registration: The study has been registered on the electronic Registry of Non-Interventional Studies (RNIS) posted on the website of the Hellenic Association of Pharmaceutical Companies (SFEE): https://www.dilon.sfee.gr/ studiesp_d.php?meleti_id=NIS-OGR-XXX-2012/1

Keywords: Metastatic breast cancer, Hormone receptor, Human epidermal growth factor receptor 2, Treatment patterns, Progression-free survival, Overall survival, Greece

\section{Background}

Breast cancer is the most frequently diagnosed cancer worldwide, conferring 523,000 deaths and 15.1 million disability-adjusted life-years in women in 2015 [1]. The estimated age-standardized incidence and mortality rate of breast cancer among females in Greece for 2012, was 58.6 and 21.0 per 100,000 , respectively, thus, being the leading cause of death from cancer among Greek women [2].

The past two decades have witnessed significant advances in awareness, screening and molecular understanding of breast cancer. Nevertheless, $6-10 \%$ of all women still present with distant and 30\% with regional lymph node metastases [3]. Additionally, an estimated $20-50 \%$ of women diagnosed with early stage breast cancer will eventually develop metastatic disease $(\mathrm{MBC})[4,5]$. While the 5 -year survival rate for patients diagnosed with localized disease is $98.8 \%$, the rate drops to $85.2 \%$ among women diagnosed with regional and to $26.3 \%$ for those diagnosed with distant metastases [3]. Median overall survival (OS) in the MBC setting is 2 to 3 years [6-8]. Age at diagnosis is considered one of the main prognostic factors of survival from MBC [9]. Additionally, patient comorbidities and menopausal status, tumor histology and pathology, hormone receptor (HR) and human epidermal growth factor receptor 2 (HER2) status, sites of metastatic involvement, number of involved axillary lymph nodes and de novo metastatic disease presentation are also considered prognostic factors of survival and treatment response [9-11]. HR and HER2 are key elements guiding selection of an individualized treatment strategy. Additional factors, taken into consideration when deciding on the optimal treatment, include the length of disease-free interval since primary diagnosis, presence of visceral crisis, menopausal status, patient preference and prior treatments with special challenges posed by the development of endocrine or anti-HER2 resistance $[9,10,12,13]$.

Evidence regarding the clinical management and outcomes of MBC in Greece largely stems from registries including patients participating in clinical trials, and to a lesser extent from studies conducted in the routine care $[7,14,15]$. This retrospective cohort study aimed to provide a snapshot of $\mathrm{MBC}$ disease burden, clinical course and healthcare resource utilization as well as to depict the management patterns in relation to HR and HER2 status in a representative population of $\mathrm{MBC}$ patients treated under real life clinical conditions in Greece.

\section{Methods}

\section{Study design and setting}

"EMERGE" was a multicenter, national, retrospective cohort study. Patient data abstraction was mainly carried out through medical chart review, but also from databases developed and maintained by co-operative groups for their research activities. As a prerequisite, the selected databases contained an adequate number of potential candidates that met the inclusion/exclusion criteria, and captured only non-identifiable patient information including the study variables. All required information for the purposes of the study was collected using paper case report forms. Chemotherapy and endocrine therapy (ET) for MBC management have been grouped using the Anatomical Therapeutic Chemical Drug Classification dictionary by the World Health Organization Collaborating Centre for Drug Statistics Methodology 'chemical subgroup', while targeted therapies (TT) have been presented as 'anti-HER2 agents', 'antiangiogenic agents' and 'mTOR inhibitor'. Supportive therapies (other than radiotherapy), such as bisphosphonates have not been captured in the context of this study.

Site selection was carried out through a documented and constructed feasibility assessment process that accounted, among others, for the expertise of the investigators in clinical study conduct, their intent to comply with the study procedures and their ability to enroll the pre-specified number of patients, as well as for the availability of patients' complete medical records. The study was conducted in accordance with the Declaration of Helsinki and all applicable local requirements.

\section{Study population}

According to the final study protocol eligible patients were comprised of females aged $\geq 18$ years, diagnosed with MBC between 01-Janaury-2010 and 30-June-2012, either de novo or having progressed from a non-metastatic state. Patients with a history of concurrent or other primary malignancies (except curatively resected non-melanoma skin cancer or in situ cervical cancer) were excluded from the study. 


\section{Study objectives and endpoints}

The primary endpoint of the study was the estimation of disease progression incidence rate (IR) per patient-year, for MBC patients on first line treatment. The secondary objectives of the study were to estimate the overall survival (OS), the progression-free survival (PFS) and the time to progression (TTP) and to estimate the overall all-cause mortality and MBC-related mortality rate. Furthermore, the study aimed to record clinical and pathological characteristics of newly diagnosed MBC patients and to describe the management patterns of $\mathrm{MBC}$, as well as the healthcare utilization associated with the disease, in Greece.

\section{Statistical methods}

The disease progression IR for patients receiving first line treatment, expressed in patient-years has been calculated by dividing the number of patients with progression in the first line setting by the overall time of follow-up (i.e. the sum of first line treatment duration of each patient). The Kaplan-Meier method has been used, in order to assess the OS, PFS and TTP [16]. PFS time has been defined as the time from first line treatment onset to the first documented disease progression or death due to any cause during the patients' overall look-back period. Differences in the OS and PFS between subgroups have been examined by the log-rank test. Hazard ratios (95\% confidence interval) between subgroups of interest have been derived by univariate Cox regression analysis. Likewise, a multivariable Cox proportional hazard model has been used to examine the association of PFS time with diagnosis of MBC de novo, presence of liver and bone metastases, receipt of endocrine therapy in the first line setting, HR and HER2 status, age at MBC diagnosis and MBC duration at first line treatment onset. Mortality rate, hospitalization as well as the emergency room and hospital outpatient visit rates (per patient-year) have been calculated by dividing the number of events by the sum of person-time (in years) of patient observation since $\mathrm{MBC}$ diagnosis. No imputation of missing data has been performed with the exception of partial dates.

Sample size evaluation was based on the maximum acceptable margin error that has been set at less than $5 \%$. Under this consideration, a sample size of 400 patients, with a significance level $\mathrm{a}=0.05$ and power $80 \%$, provided a margin of error of no more than 0.049 . All statistical tests were two-sided and were performed at a 0.05 significance level. Statistical analysis has been conducted using SAS $^{\bullet}$ v9.3 (SAS Institute, Cary, NC).

\section{Results}

\section{Patient characteristics}

A total of 386 patients diagnosed with MBC between 8-January-2010 and 27-June-2012 were enrolled in the study from 12-March-2013 to 31-March-2015, by oncologists practicing in 16 hospital-based sites distributed in 4 regions of Greece. The 12 sites located in Attica enrolled $77.5 \%$ of the patients. Of the enrolled patients, 7 did not meet all inclusion criteria. The median study look-back period of the eligible population was 29.1 [interquartile range (IQR): 19.2-37.7] months.

Eligible patients $(N=379)$ had been diagnosed with $\mathrm{MBC}$ at a mean $\pm \mathrm{SD}$ age of $60.4 \pm 12.8$ years. At MBC diagnosis, $77.6 \%(256 / 330)$ were postmenopausal, $81.4 \%$ $(206 / 253)$ had at least one term pregnancy and $54.8 \%$ $(119 / 217)$ had a positive nursing history, while $92.0 \%$ $(344 / 374)$ had an ECOG PS of 0 or 1 . The primary tumor was histologically classified as invasive ductal carcinoma (IDC) in $76.0 \%$ of patients, as invasive lobular carcinoma in $7.9 \%$, and for the remaining patients the tumor had characteristics of IDC with other histological features. The primary tumor differentiation was grade I or I/II in $3.4 \%$, grade II or III or II/III in $81.5 \%$, while for $15.0 \%$ the grade was unknown. The most common metastatic sites were the bones $(207 / 369 ; 56.1 \%)$, lungs (138/369; 37.4\%;) and liver (28.7\%; 106/369) (Table 1).

\section{Patient disposition based on HR/HER2 status and on de novo diagnosis or progression from an earlier stage}

At the time of $\mathrm{MBC}$ diagnosis, nearly half of the patients (49.9\%; 189/379) were $\mathrm{HR}^{+} / \mathrm{HER} 2^{-}$, while $14.8 \%$ (56/ 379) were $\mathrm{HR}^{+} / \mathrm{HER} 2^{+}, 12.9 \%$ (49/379) triple negative, and $11.3 \%(43 / 379) \mathrm{HR}^{-} / \mathrm{HER}^{+}$. Classification could not be performed in the remaining patients $(11.1 \% ; 42 / 379)$. Additionally, 59.9\% (227/379) of the eligible population had been first diagnosed at an earlier breast cancer stage (a mean \pm SD of $5.2 \pm 5.3$ years prior to MBC diagnosis), while the remaining $40.1 \%$ (152/379) presented with de novo metastatic disease.

\section{MBC management patterns}

Of the eligible patients $(n=379), 99.5 \%$ had been exposed to systemic therapy, $32.2 \%$ had received radiotherapy and $13.2 \%$ had undergone surgery for MBC management. Of the patients exposed to systemic treatment $(n=377), 374$ had received first line therapy [median exposure 5.5 (IQR: 3.7-11.0) months], 254 proceeded to second line [median 4.4 (IQR: 2.7-7.9) months], 175 to third line [median 3.5 (IQR: 1.9-5.7) months] and 105 to fourth line treatment. Information for patients of each subpopulation per HR/ HER2 status that received first line treatment and those that advanced to the second, third and beyond the third line is indicated in Table 2.

The median time elapsed from $\mathrm{MBC}$ diagnosis to first line treatment onset was 0.6 (IQR: 0.2-1.4) months (373 patients with available data). Chemotherapy was administered in $76.7 \%$ (287/374) of the overall population in the first line setting; TT (i.e. anti-HER2 agents, antiangiogenic 
Table 1 Clinical and primary tumor characteristics of the overall population at MBC diagnosis $(N=379)$

\begin{tabular}{|c|c|c|}
\hline \multicolumn{2}{|l|}{ Characteristic } & \multirow{2}{*}{$\frac{N(\%)}{371(99.2)}$} \\
\hline Caucasians & & \\
\hline Median age at MBC diagnosis, years & & $61.2(50.7-70.9)$ \\
\hline $\mathrm{BMI}$ at $\mathrm{MBC}$ diagnosis $\left(\mathrm{kg} / \mathrm{m}^{2}\right)$, median $(\mathrm{IQR})(N=320)$ & & $27.0(23.9-31.4)$ \\
\hline \multirow[t]{4}{*}{ BMI category at MBC diagnosis $(N=320)$} & Underweight $\left(B M I<18.5 \mathrm{~kg} / \mathrm{m}^{2}\right)$ & $2(0.6)$ \\
\hline & Normal $\left(B M I \geq 18.5\right.$ and $\left.B M l<25 \mathrm{~kg} / \mathrm{m}^{2}\right)$ & $117(36.6)$ \\
\hline & Overweight (BMI $\geq 25$ and $\left.B M I<30 \mathrm{~kg} / \mathrm{m}^{2}\right)$ & $102(31.9)$ \\
\hline & Obese $\left(B M I \geq 30 \mathrm{~kg} / \mathrm{m}^{2}\right)$ & 99 (30.9) \\
\hline \multirow[t]{3}{*}{ Menopausal status at MBC diagnosis $(N=330)$} & Postmenopausal & $256(77.6)$ \\
\hline & Premenopausal & $66(20.0)$ \\
\hline & Perimenopausal & $8(2.5)$ \\
\hline \multicolumn{2}{|l|}{ Use of oral contraceptives $(N=236)$} & $9(3.8)$ \\
\hline \multicolumn{2}{|l|}{ Use of hormone replacement therapy $(N=238)$} & $10(4.2)$ \\
\hline \multicolumn{2}{|l|}{ Family history of breast cancer $(N=323)$} & $47(14.6)$ \\
\hline \multicolumn{2}{|l|}{ Family history of ovarian cancer $(N=322)$} & $5(1.6)$ \\
\hline \multirow[t]{5}{*}{ Comorbidities at MBC diagnosis $^{\mathrm{a}}(\mathrm{N}=379)$} & Vascular disorders & $61(16.1)$ \\
\hline & Cardiac disorders & $51(13.5)$ \\
\hline & Endocrine disorders & $50(13.2)$ \\
\hline & Psychiatric disorders & $24(6.3)$ \\
\hline & Metabolism and nutrition disorders & $22(5.8)$ \\
\hline \multirow[t]{2}{*}{ ECOG performance status at MBC diagnosis $(N=374)$} & PS $0-1$ & $344(92.0)$ \\
\hline & PS $2-3$ & $30(8.0)$ \\
\hline \multirow[t]{3}{*}{ Primary tumor location $(N=376)$} & Left breast & $189(50.3)$ \\
\hline & Right breast & $179(47.6)$ \\
\hline & Both breasts & $8(2.1)$ \\
\hline \multirow[t]{3}{*}{ Primary tumor size $(N=301)$} & Tumor size $\leq 2 \mathrm{~cm}$ & $110(36.5)$ \\
\hline & $2 \mathrm{~cm}<$ tumor size $\leq 5 \mathrm{~cm}$ & $148(49.2)$ \\
\hline & Tumor size $>5 \mathrm{~cm}$ & $43(14.3)$ \\
\hline \multicolumn{2}{|c|}{ Number of metastases at MBC diagnosis, median (IQR) $\left(N=264^{\mathrm{b}}\right)$} & $2.0(1.0-3.0)$ \\
\hline \multirow[t]{6}{*}{ Distant metastatic sites $(N=369)^{a}$} & Bones & $207(56.1)$ \\
\hline & Lung & $138(37.4)$ \\
\hline & Liver & $106(28.7)$ \\
\hline & Lymph nodes & $70(19.0)$ \\
\hline & Brain & $35(9.5)$ \\
\hline & Pleural effusion & $29(7.9)$ \\
\hline
\end{tabular}

${ }^{\mathrm{a}}$ Only those present in at least $5 \%$ of the population have been included

${ }^{b}$ Patients for whom the precise number of metastases was known

$\mathrm{IQR}$, interquartile range (Q25-Q75); N, number of patients with known data for each variable

agents and mTOR inhibitor) in 52.4\% (196/374) and ET in $28.3 \%(106 / 374)$; additionally, chemotherapy, TT and ET were administered in $65.0 \%$ (165/254), 46.5\% (118/ $254)$ and $36.6 \%(93 / 254)$ of patients in the second line; in $73.1 \%$ (128/175), $42.3 \%$ (74/175) and $28.6 \%$ (50/175) of patients in the third line setting; and in $87.6 \%$ (92/105), $42.9 \%$ (45/105), 36.2\% (38/105) of patients receiving systemic fourth line treatment and beyond.
Systemic treatment patterns in terms of therapeutic drug classes administered in the first, second and third line setting of the overall population and subpopulations per HR/HER2 status are displayed in Fig. 1. Similarly, first, second, third and beyond the third line treatment patterns per therapeutic agent(s) subgroup administered in at least $10 \%$ of any of the subpopulations per HR/ HER2 status are displayed in Table 2. 
Table 2 Systemic treatment management patterns per HR/HER2 status

\begin{tabular}{|c|c|c|c|c|c|}
\hline & Overall & $\mathrm{HR}^{+} / \mathrm{HER} 2^{-}$ & $\mathrm{HR}^{+} / \mathrm{HER} 2^{+}$ & $\mathrm{HR}^{-} / \mathrm{HER} 2^{+}$ & $\mathrm{HR}^{-} / \mathrm{HER}^{-}$ \\
\hline \multirow[t]{2}{*}{ Patients receiving first line treatment } & $N=374$ & $N=188$ & $N=56$ & $N=43$ & $N=46$ \\
\hline & n (\%) & n (\%) & n (\%) & n (\%) & n (\%) \\
\hline Taxanes \& Antiangiogenic agents & $54(14.4)$ & $35(18.6)$ & $4(7.1)$ & $1(2.3)$ & $6(13.0)$ \\
\hline Aromatase inhibitors only & $41(11.0)$ & $36(19.1)$ & $2(3.6)$ & - & $1(2.2)$ \\
\hline Platinum compounds \& Taxanes \& Antiangiogenic agents & $19(5.1)$ & $5(2.7)$ & - & - & $13(28.3)$ \\
\hline Taxanes \& Anti-HER2 agents & $18(4.8)$ & $1(0.5)$ & $11(19.6)$ & $6(14.0)$ & - \\
\hline Pyrimidine analogues \& Anti-HER2 agents & $15(4.0)$ & $2(1.1)$ & $4(7.1)$ & $9(20.9)$ & - \\
\hline Other chemotherapy \& Anti-HER2 agents & $9(2.4)$ & - & $3(5.4)$ & $6(14.0)$ & - \\
\hline \multirow[t]{2}{*}{ Patients receiving second line treatment } & $N=254$ & $N=125$ & $N=36$ & $N=35$ & $N=33$ \\
\hline & n (\%) & n (\%) & n (\%) & n (\%) & n (\%) \\
\hline Aromatase inhibitors only & $21(8.3)$ & $18(14.4)$ & $1(2.8)$ & - & $1(3.0)$ \\
\hline Anti-estrogens only & $20(7.9)$ & $17(13.6)$ & - & - & - \\
\hline Aromatase inhibitors \& mTOR inhibitors & $20(7.9)$ & $17(13.6)$ & - & - & $2(6.1)$ \\
\hline Pyrimidine analogues \& anti-HER2 agents & $20(7.9)$ & $1(0.8)$ & $7(19.4)$ & $10(28.6)$ & $2(6.1)$ \\
\hline Anti-HER2 agents only & $11(4.3)$ & $1(0.8)$ & $1(2.8)$ & $8(22.9)$ & $1(3.0)$ \\
\hline Other chemotherapy \& Anti-HER2 agents & $6(2.4)$ & - & $2(5.6)$ & $4(11.4)$ & - \\
\hline Platinum compounds \& Pyrimidine analogues & $6(2.4)$ & $1(0.8)$ & - & - & $4(12.1)$ \\
\hline Aromatase inhibitors \& Anti-HER2 agents & $6(2.4)$ & - & $6(16.7)$ & - & - \\
\hline \multirow[t]{2}{*}{ Patients receiving third line treatment } & $N=175$ & $N=89$ & $N=28$ & $N=24$ & $N=18$ \\
\hline & n (\%) & n (\%) & n (\%) & n (\%) & n (\%) \\
\hline Taxanes only & $15(8.6)$ & $12(13.5)$ & $1(3.6)$ & $1(4.2)$ & $1(5.6)$ \\
\hline Pyrimidine analogues \& Anti-HER2 agents & $13(7.4)$ & - & $4(14.3)$ & $8(33.3)$ & $1(5.6)$ \\
\hline Pyrimidine analogues only & $11(6.3)$ & $10(11.2)$ & $1(3.6)$ & - & - \\
\hline Aromatase inhibitors only & $11(6.3)$ & $10(11.2)$ & - & - & - \\
\hline Anthracyclines \& Other chemotherapy & $7(4.0)$ & $4(4.5)$ & - & $1(4.2)$ & $2(11.1)$ \\
\hline Other chemotherapy \& Anti-HER2 agents & $6(3.4)$ & $1(1.1)$ & $3(10.7)$ & $1(4.2)$ & $1(5.6)$ \\
\hline Pyrimidine analogues \& Antiangiogenic agents & $5(2.9)$ & $2(2.2)$ & - & - & $3(16.7)$ \\
\hline Anti-HER2 agents only & $5(2.9)$ & - & - & $5(20.8)$ & - \\
\hline \multirow[t]{2}{*}{ Patients receiving beyond the third line treatment } & $N=105$ & $N=49$ & $N=17$ & $N=18$ & $N=11$ \\
\hline & n (\%) & n (\%) & n (\%) & n (\%) & n (\%) \\
\hline Pyrimidine analogues only & $6(5.7)$ & $5(10.2)$ & $1(5.9)$ & - & - \\
\hline Anti-estrogens only & $5(4.8)$ & $5(10.2)$ & - & - & - \\
\hline Anthracyclines \& Anti-HER2 agents & $3(2.9)$ & - & - & $2(11.1)$ & $1(9.1)$ \\
\hline Other chemotherapy \& Anti-HER2 agents & $3(2.9)$ & - & - & $3(16.7)$ & - \\
\hline Pyrimidine analogues \& Anti-HER2 agents & $2(1.9)$ & - & - & $2(11.1)$ & - \\
\hline Pyrimidine analogues \& Other chemotherapy \& Anti-HER2 agents & $2(1.9)$ & - & - & $2(11.1)$ & - \\
\hline Pyrimidine analogues \& Taxanes \& Other chemotherapy & $2(1.9)$ & - & - & - & $2(18.2)$ \\
\hline
\end{tabular}

Only systemic treatment patterns utilized in at least $10.0 \%$ of one or more of the four subpopulations per HR/HER2 status are shown. Taxanes (docetaxel, paclitaxel, nab-paclitaxel); platinum compounds (carboplatin, cisplatin); pyrimidine analogues (capecitabine, fluorouracil, gemcitabine); anthracyclines (doxorubicin, liposomal and pegylated doxorubicin, epirubicin); aromatase inhibitors (anastrozole, exemestane, letrozole); anti-estrogens (tamoxifen, fulvestrant); other chemotherapy (cyclophosphamide, eribulin, methotrexate, vinorelbine); anti-HER2 agents (trastuzumab, lapatinib); antiangiogenic agents (bevacizumab, sorafenib), mTOR inhibitor (everolimus). N, number of patients analyzed for each outcome; $n$, number of patients who received the particular treatment 


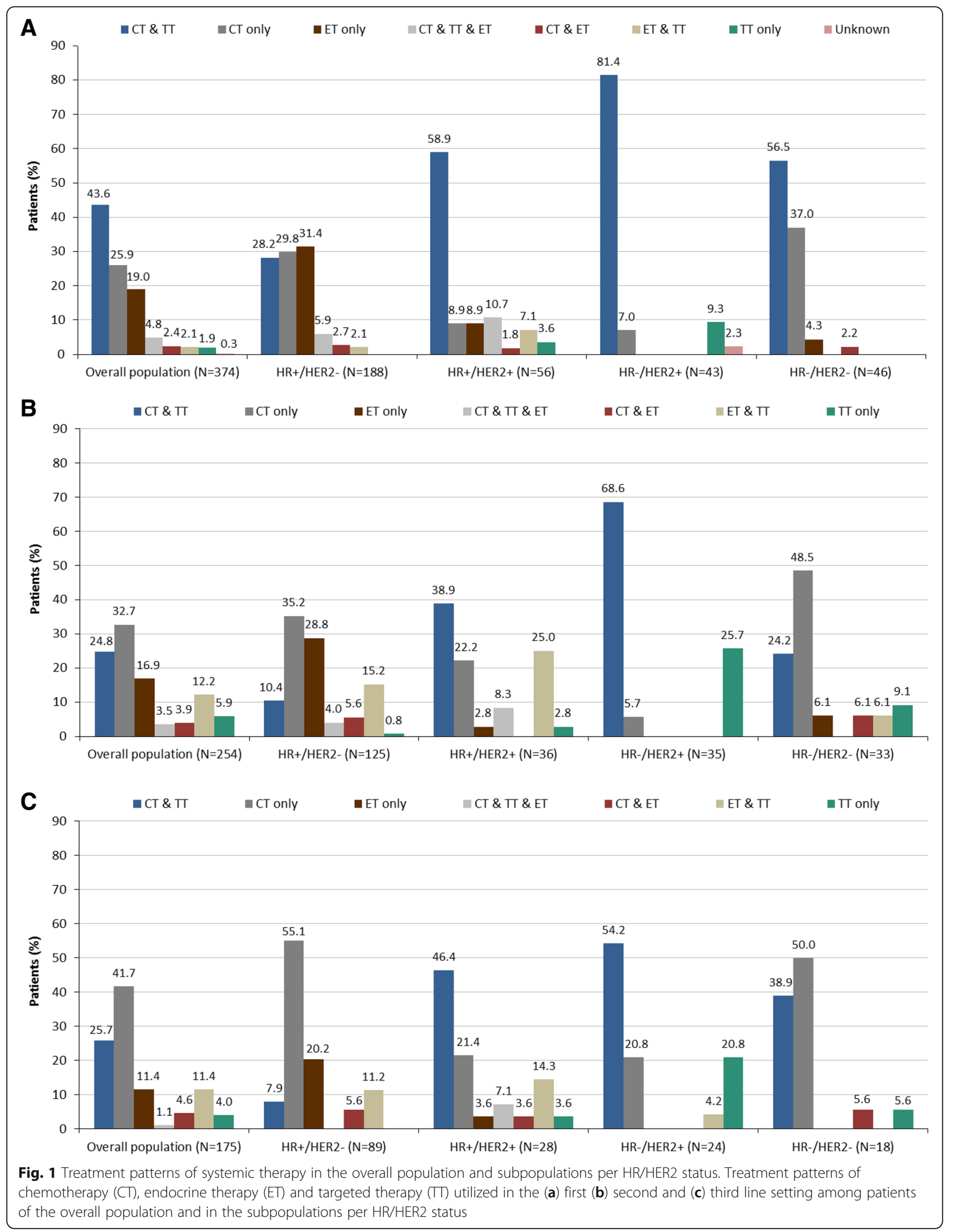


In regards to the treatment patterns (as displayed in Fig. 1) utilized from the first to the second and third line of therapy in the overall population, no single dominant pattern emerged. It shall be noted that a total of $30.2 \%$ (57/ 189), $25.0 \%$ (14/56), 58.1\% (25/43) and 34.7\% (17/49) of the $\mathrm{HR}^{+} / \mathrm{HER} 2^{-}, \mathrm{HR}^{+} / \mathrm{HER} 2^{+}, \mathrm{HR}^{-} / \mathrm{HER} 2^{+}$and $\mathrm{HR}^{-} / \mathrm{HER}^{-}$ patients had received radiotherapy in the MBC setting.

\section{Clinical outcomes: Disease progression incidence rate,} progression-free survival, overall survival and mortality rate The disease progression IR in the overall population over a cumulative 256.7 years of follow-up in the first line setting, was 0.57 (95\% CI: 0.48-0.67) per person-year. The IR was the lowest among $\mathrm{HR}^{+} / \mathrm{HER}^{-}$(0.47; 95\% CI: $0.37-$ $0.58)$ patients. Additionally, the IR was higher among patients primarily diagnosed at an earlier stage $(0.85 ; 95 \%$ CI: $0.69-1.01)$ rather than de novo metastatic $(0.29 ; 95 \%$ CI: 0.20-0.38) (Table 3).

The Kaplan-Meier estimated median PFS of the overall population over the study observation period was 22.4 months (95\% CI: 20.4-24.7) while the median TTP was 22.8 months (95\% CI: 20.8-25.2). The estimated median PFS in the subpopulation primarily diagnosed at an earlier stage was shorter than that of patients diagnosed with de novo MBC (log-rank $p$-value: 0.004) (Fig. 2A and Table 3). A statistically significant difference was also detected in the median PFS of the subpopulations per HR/HER2 status (log-rank: p-value $=0.040$ ) (Fig. 2B). Specifically, the median PFS of the $\mathrm{HR}^{+} / \mathrm{HER}^{+}$subpopulation was 19.7 months (95\% CI: $12.9-25.9$ ) and that of the $\mathrm{HR}^{-} / \mathrm{HER} 2^{-} 18.3$ months (95\% CI: 10.0-24.7), both shorter than that of the $\mathrm{HR}^{+} /$ HER2 ${ }^{-}$subpopulation (24.6 months, 95\% CI: 21.3-27.9).

Among the factors (Table 4) examined by a multivariable Cox proportional hazards model in regards to their association with disease progression, median PFS was estimated to be higher among patients receiving ET as part of the first line treatment [HR: 0.70 (95\% CI: 0.51-0.95; $p=0.024)$, and lower in patients first diagnosed at an earlier breast cancer stage [HR: 1.42 (95\% CI: 1.09-1.85; $p=0.009)]$ as well as among those with liver metastases [HR: 1.45 (95\% CI: 1.10-1.93); $\mathrm{p}=0.009$ ].

In regards, to the all-cause mortality rate, 143 deaths (37.7\% of the overall population) due to any cause were recorded over a cumulative study observation period of 905.0 years, yielding a rate of 0.16 per person-year. The respective $\mathrm{MBC}$-related mortality rate was 0.15 per person-year. The Kaplan-Meier estimated median OS was 45.0 months (95\% CI: 40.9-55.0) (Table 3). The relative risk of death did not significantly differ between patients first diagnosed at an earlier breast cancer stage vs. those with a diagnosis of de novo MBC (Fig. 3A and Table 3). The relative risk of death was significantly higher for $\mathrm{HR}^{-} / \mathrm{HER}^{+}$vs. $\mathrm{HR}^{+} / \mathrm{HER} 2^{-}$and for triple negatives vs. $\mathrm{HR}^{+} / \mathrm{HER}^{-}{ }^{-}$[HR: 1.71 (95\% CI: $1.05-2.78$; $p=0.032$ ); and HR: 1.79 (95\% CI: 1.12-2.87; $p=0.015$ ), respectively] (Fig. 3B and Table 3).

\section{Healthcare resource utilization}

Over a cumulative observation period of 660.5 years, the all-cause hospitalization incidence rate was 0.74 per person-year. The hospitalization incidence rate due to treatment-related toxicity and due to other reasons was 0.21 (cumulative observation period: 724.0 years) and 0.51 (cumulative observation period: 652.6 years) per person-year, respectively.

Similarly, the all-cause emergency room and hospital outpatient visit rate over a cumulative follow-up of 605.4 years was 2.67 per person-year. The emergency room and hospital outpatient visit rate for treatment-related toxicity was 0.58 (cumulative observation period: 638.1 years), while the respective rate due to other reasons was 2.02 (cumulative observation period: 618.3 years).

\section{Discussion}

This study has yielded evidence on the clinicopathological characteristics, management patterns and treatment outcomes in the real-life clinical practice of Greece of $379 \mathrm{MBC}$ patients. In addition to the overall population, treatments and clinical outcomes have been presented in the subpopulations diagnosed de novo and having progressed from a non-metastatic state and in the four subpopulations by joint HR and HER2 status. Although microarray gene-expression profiling constitutes a promising tool for prognostication and disease management, HR and HER2 status remains widely used for guiding treatment decision in the routine care $[9,10,17,18]$. Overall, of the $337 \mathrm{MBC}$ patients with known HR/HER2 status enrolled in the study, 56\% were $\mathrm{HR}^{+} / \mathrm{HER} 2^{-}$, while a nearly equal percentage were $\mathrm{HR}^{+} / \mathrm{HER}^{+}(17 \%)$ and triple negative (15\%), matching the distribution reported elsewhere $[18,19]$.

The disease progression IR in the overall population was 0.57 (95\% CI: 0.48-0.67) per person-year; the median OS was 45.0 months, i.e. higher than the general estimates of about 2 years reported elsewhere [6-8], but similar (44 months) to that reported in a study of similar design conducted in a single institution in Germany [20]. The lowest disease progression IR and the longest median PFS and OS were observed in $\mathrm{HR}^{+} / \mathrm{HER}^{-}$patients, in alignment with other studies demonstrating a better prognosis for this breast cancer entity [5]. Despite the fact that the guideline-recommended treatment of choice for $\mathrm{HR}^{+} / \mathrm{HER}^{-}$disease is sequential ET, only $42 \%$ of the subpopulation in the present study had received ET-containing first line therapy and the remaining 58\% had received chemotherapy-based regimens (with or without TT). Of further concern is the 


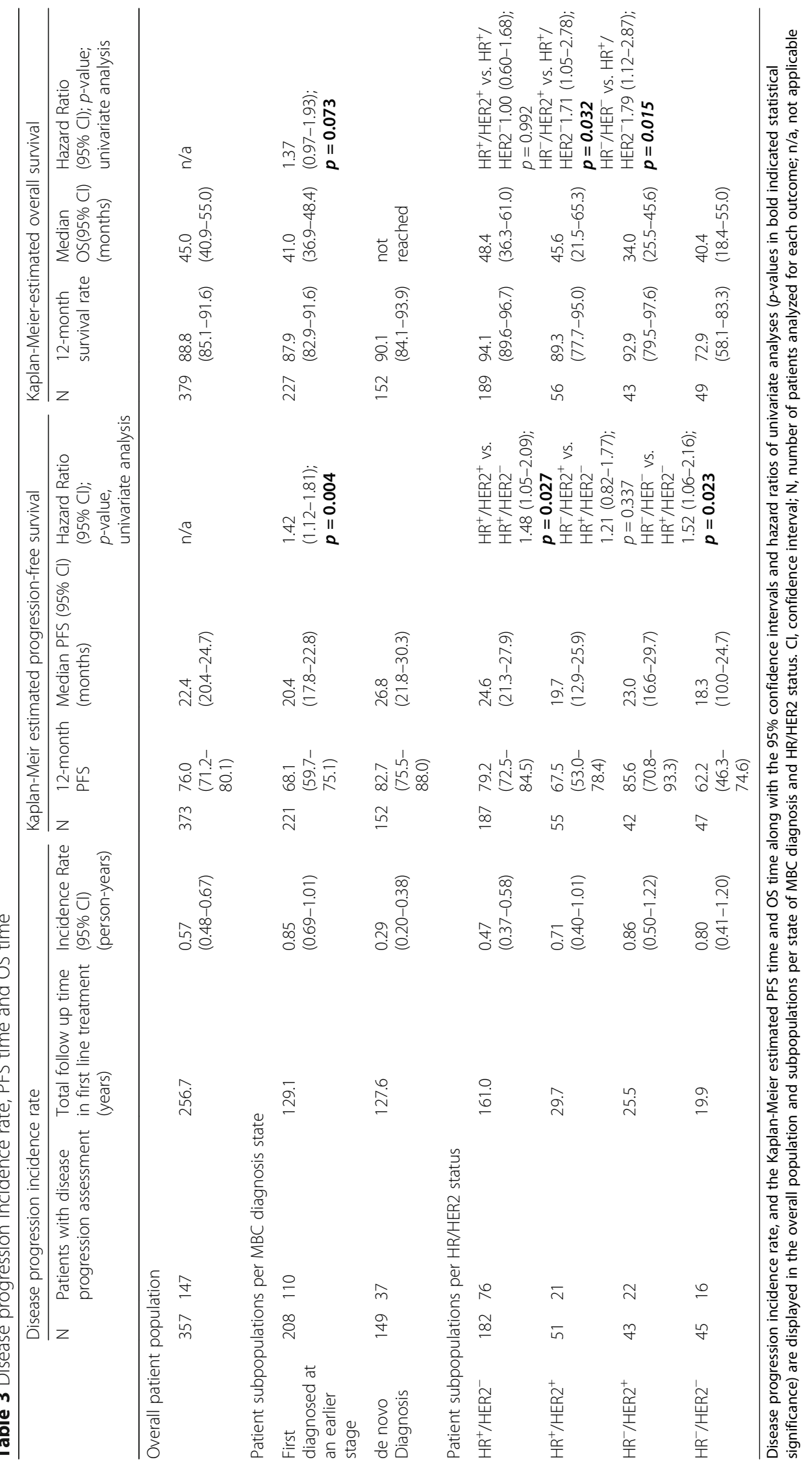


A

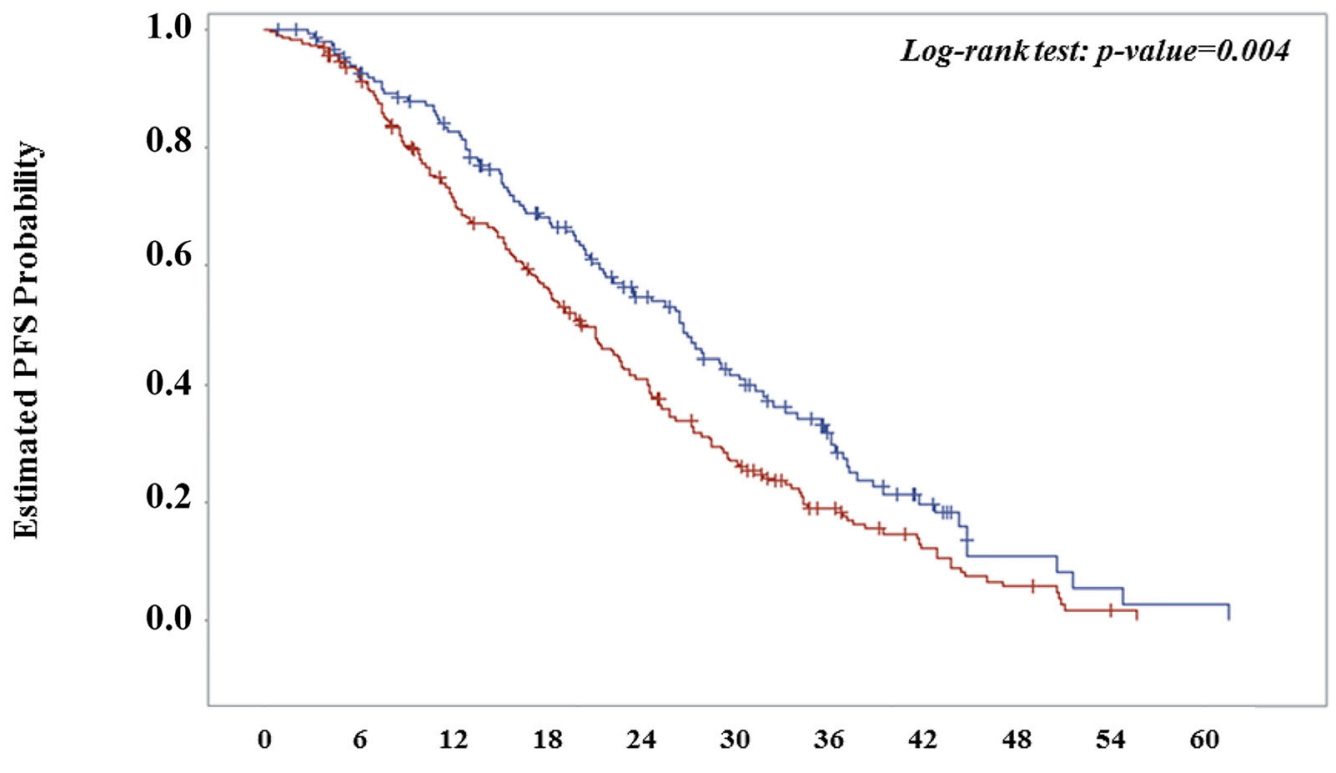

People at risk

Time (months)

$\begin{array}{lccccccccccc}\text { De novo } & 152 & 137 & 116 & 90 & 65 & 46 & 29 & 13 & 4 & 2 & 1 \\ \begin{array}{l}\text { First diagnosed at an } \\ \text { earlier stage }\end{array} & 221 & 201 & 151 & 116 & 81 & 51 & 28 & 15 & 7 & 2 & 0\end{array}$

B

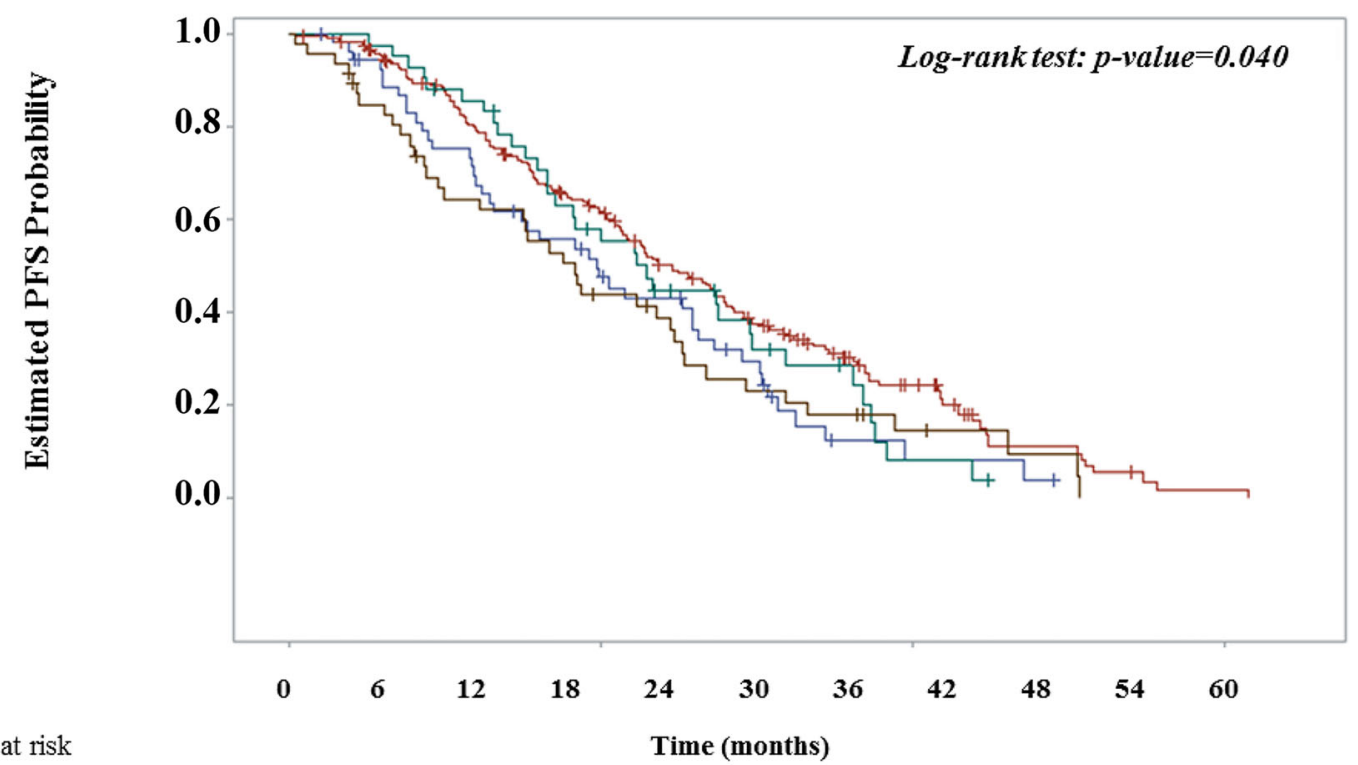

People at risk

$\begin{array}{rrrrrrrrrrr}55 & 48 & 36 & 28 & 20 & 12 & 3 & 2 & 1 & 0 & \\ 187 & 173 & 140 & 110 & 80 & 58 & 37 & 19 & 8 & 4 & 1 \\ 42 & 41 & 35 & 25 & 16 & 10 & 7 & 2 & 0 & & \\ 47 & 38 & 28 & 22 & 15 & 9 & 7 & 3 & 2 & 0 & \end{array}$

Fig. 2 Kaplan-Meier progression-free survival (PFS) plots. PFS plots and the relevant log-rank test $p$-value is shown for MBC patients (a) diagnosed de novo versus at an earlier stage and (b) per HR/HER2 status 
Table 4 Multivariable Cox proportional hazard model to identify factors associated with PFS in the overall population

\begin{tabular}{|c|c|c|c|}
\hline Multivariable Cox proportional hazard model $(N=319)$ & Hazard ratio & 95\% confidence interval & $p$-value \\
\hline First diagnosed at an earlier stage vs. de novo MBC diagnosis & $1.42^{\mathrm{a}}$ & $1.09-1.85$ & 0.009 \\
\hline Presence of liver metastases vs. No liver metastases & $1.45^{\mathrm{b}}$ & $1.10-1.93$ & 0.009 \\
\hline Presence of bone metastases vs. No bone metastases & $0.91^{\mathrm{c}}$ & $0.69-1.20$ & 0.509 \\
\hline Receipt of endocrine therapy vs. No endocrine therapy as part of first line treatment & $0.70^{\mathrm{d}}$ & $0.51-0.95$ & 0.024 \\
\hline Negative vs. Positive HER2 status & $0.91^{e}$ & $0.68-1.22$ & 0.531 \\
\hline Positive vs. Negative HR status & $1.01^{\mathrm{f}}$ & $0.74-1.37$ & 0.970 \\
\hline Age at MBC diagnosis (years) & $1.00^{9}$ & $0.99-1.01$ & 0.953 \\
\hline Time from MBC diagnosis to first line treatment (months) & $0.97^{\mathrm{h}}$ & $0.93-1.01$ & 0.154 \\
\hline
\end{tabular}

${ }^{2}$ Adjusted for presence of liver and bone metastases, receipt of endocrine therapy in the first line setting, HR and HER2 status, age at MBC diagnosis, and MBC duration at first line treatment onset

${ }^{\mathrm{b}}$ Adjusted for diagnosis of MBC de novo, presence of bone metastases, receipt of endocrine therapy in the first line setting, HR and HER2 status, age at MBC diagnosis, and $\mathrm{MBC}$ duration at first line treatment onset

'Adjusted for diagnosis of MBC de novo, presence of liver metastases, receipt of endocrine therapy in the first line setting, HR and HER2 status, age at MBC diagnosis, and $\mathrm{MBC}$ duration at first line treatment onset

${ }^{\mathrm{d}}$ Adjusted for diagnosis of MBC de novo, presence of liver and bone metastases, HR and HER2 status, age at MBC diagnosis, and MBC duration at first line treatment onset

${ }^{e}$ Adjusted for diagnosis of $M B C$ de novo, presence of liver and bone metastases, receipt of endocrine therapy in the first line setting, HR status, age at MBC diagnosis, and MBC duration at first line treatment onset

${ }^{f}$ Adjusted for diagnosis of MBC de novo, presence of liver and bone metastases, receipt of endocrine therapy in the first line setting, HER2 status, age at MBC diagnosis, and $\mathrm{MBC}$ duration at first line treatment onset

${ }^{9}$ Adjusted for diagnosis of MBC de novo, presence of liver and bone metastases, receipt of endocrine therapy in the first line setting, HR and HER2 status, and MBC duration at first line treatment onset

${ }^{\mathrm{h}}$ Adjusted for diagnosis of MBC de novo, presence of liver and bone metastases, receipt of endocrine therapy in the first line setting, HR and HER2 status, and age at MBC diagnosis

fact that herein only $54 \%$ received ET in the second line setting. Suboptimal rates of ET in $\mathrm{HR}^{+} / \mathrm{HER} 2^{-}$patients have also been reported from data from US databases covering the decade of 2002-2012, with $60 \%$ receiving ET in the first line, and about three quarters not receiving a second ET [21]. Collectively, these observations suggest that in the real-world practice of Greece, chemotherapy is favored over $\mathrm{ET}$ in $\mathrm{HR}^{+} / \mathrm{HER} 2^{-}$patients, despite the fact that as demonstrated in this and other studies [10, 22], ET was shown to be associated with longer PFS. The factors contributing to the administration of chemotherapy over ET cannot be inferred, since the characteristics of this subpopulation have not been examined separately, and the presence of visceral crisis, the only condition under which the guidelines recommend administration of chemotherapy $[9,10]$, was not collected. Clarification of the reasons for this divergence from the guidelines in the routine care of Greece would be an interesting topic for further study.

In regards to triple negative cancer, the present study also supports reports that this subpopulation exhibits the worst clinical outcomes [23, 24]. Treatment for nearly all triple negative patients was comprised of chemotherapeutic regimens ( $96 \%$ in the first line setting), while paradoxically about $4 \%$ in the first line setting and $6 \%$ in the second line setting were exclusively treated with ET.

Furthermore, even though most studies indicate that among patients overexpressing HER2, survival and disease-free interval is better among those with a positive versus a negative HR status $[25,26]$, in the present study no statistical significance in the clinical outcomes of the two groups could be established, likely due to the relatively small number of patients in these two groups. In line with the evidence-based management guidelines [10], TT was used in the majority of patients overexpressing HER2; specifically, $80 \%$ of the triple positive and $93 \%$ of the $\mathrm{HR}^{-} / \mathrm{HER} 2^{+}$subpopulation had received $\mathrm{TT}$ as part of their first line therapy.

In addition, the present study adds to the observations that patients diagnosed de novo compared to those that progressed from an earlier disease state have better clinical outcomes (PFS and OS). Importantly, the survival advantage of de novo MBC patients compared to those with recurrence from an earlier stage has been linked to a short disease-free interval $(\leq 24$ months in one study [8], and $<5$ years in another [11]). Furthermore, in the present study, the most common sites of metastasis were the bones, followed by the lungs, liver and brain, in line with other reports $[27,28]$. Herein, the presence of liver metastasis, but not bone metastases, was shown to be associated with a shorter PFS confirming once again the perception that liver involvement in MBC signals the end of the natural course of the disease, while, on the other hand, bone involvement points out a more indolent disease course [28].

Apart from the study limitations inherent in its retrospective design, the following limitations should be acknowledged. First, for about $11 \%$ of the patients the HR/ HER2 status was unclassified, due mainly to unknown HER2 status; as a result a bias of unknown magnitude 
A

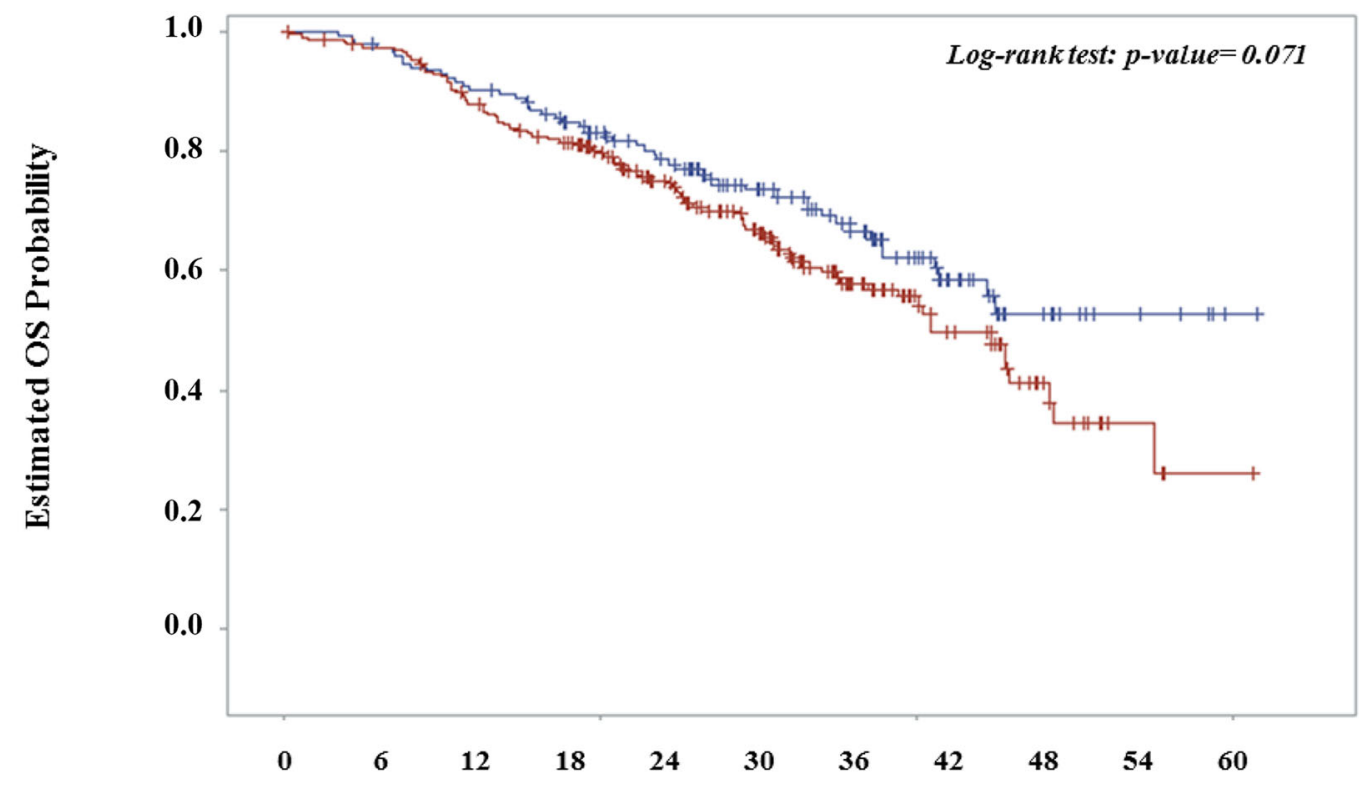

People at risk

Time (months)

$\begin{array}{lccccccccccc}\text { De novo } & 152 & 147 & 136 & 120 & 100 & 77 & 53 & 27 & 13 & 6 & 1 \\ \begin{array}{l}\text { First diagnosed at an } \\ \text { earlier stage }\end{array} & 227 & 218 & 195 & 177 & 139 & 105 & 56 & 30 & 14 & 4 & 1\end{array}$

B

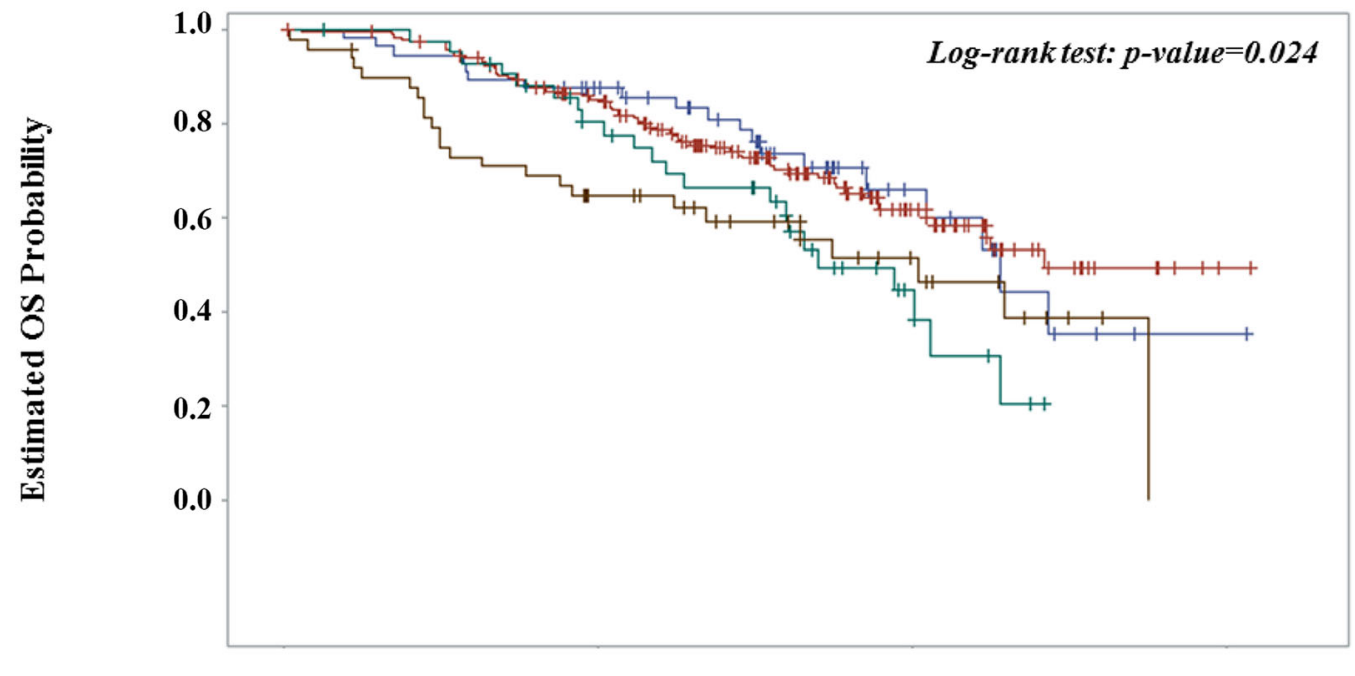

People at risk

$\begin{array}{lllllllllll}0 & 6 & 12 & 18 & 24 & 30 & 36 & 42 & 48 & 54 & 60\end{array}$

$\begin{array}{lccccccccccc}\text { HR+/HER2+ } & 56 & 54 & 50 & 48 & 39 & 32 & 17 & 10 & 5 & 2 & 1 \\ \text { HR+/HER2- } & 189 & 186 & 174 & 151 & 127 & 97 & 60 & 32 & 15 & 6 & 1 \\ \text { HR-/HER2+ } & 43 & 42 & 39 & 34 & 26 & 22 & 11 & 4 & 1 & 0 & \\ \text { HR-/HER2- } & 49 & 43 & 35 & 32 & 25 & 19 & 13 & 7 & 4 & 1 & 0\end{array}$

Fig. 3 Kaplan-Meier overall survival (OS) plots. OS plots and the relevant log-rank test $p$-value is shown for MBC patients (a) diagnosed de novo versus at an earlier stage and (b) per HR/HER2 status 
and direction may have been introduced in the patient distribution per HR/HER2 status. Second, healthcare utilization was unknown for a relatively large number of patients (approximately 21\% in regards to hospitalizations and $37 \%$ for emergency room and outpatient visits). Third, recording of management patterns did not extend to the utilization of supportive therapies, such as bisphosphonates, but are restricted to radiotherapy, chemotherapy, endocrine and targeted therapies in the MBC setting. In addition, regarding PFS and TTP, it should further be noted that due to the observational retrospective nature of the study, no specific response criteria or schedule of response assessments were dictated by the protocol; therefore, tumor progression assessment may have been performed less frequently than it would be in a clinical trial setting, delaying identification of disease progression, which could have led to an upward bias of these outcomes. Notably, this inherent limitation of real-world PFS hinders comparability with data generated in other studies. Furthermore, due to the large number of censored data in the OS analysis should be taken into consideration when appraising the robustness of the estimated median OS of the overall population. In contrast, the strengths of the present study lie in its medical chart review design which aided the depiction of normal clinical practice settings under real-life conditions, and in enrollment of the study population by 16 study sites geographically distributed across four regions of Greece, which allows for variations in local standards of care to be reflected.

\section{Conclusions}

In conclusion, "EMERGE" has yielded clinically-relevant real-world data for a diverse patient population with a disease of complex and heterogeneous biology, such as MBC. The findings clearly demonstrate differences between the subtypes per HR/HER2 status in clinical outcomes and mortality and identify areas of divergence from evidence-based guideline recommendations for $\mathrm{MBC}$ management, especially as it pertains to the $\mathrm{HR}^{+} / \mathrm{HER} 2^{-}$subpopulation. In order to achieve the maximum benefit from existing, but also from promising novel treatment options for breast cancer management, further studies that specifically examine the factors guiding the physician's treatment decision-making in routine care, should be considered.

\footnotetext{
Abbreviations

Cl: confidence interval; ET: endocrine therapy; HER2: human epidermal growth factor receptor 2; HR: hormone receptor; IDC: invasive ductal carcinoma; IQR: interquartile range; IR: incidence rate; MBC: metastatic breast cancer; OS: overall survival; PFS: progression-free survival; TT: targeted therapy; TTP: time to progression
}

\section{Funding}

The study was sponsored by AstraZeneca Greece. The study Sponsor was involved in the study design, but not in the collection, analysis or interpretation of the data included in this publication. AstraZeneca also funded the medical writing support for this publication.

\section{Availability of data and materials}

The full clinical study report supporting the data and conclusions of this article is available on the Hellenic electronic Registry of Non-Interventional Studies and can be downloaded from: https://www.dilon.sfee.gr/studiesp_d.php?meleti_id=NIS-OGR-XXX-2012/1. Row Datasets are available from the authors upon reasonable request and with permission of the sponsor.

\section{Authors' contributions \\ AK, AA, GK, ES, and AP have made substantial contributions to acquisition and interpretation of the data and were involved in revising the manuscript critically for important intellectual content. Furthermore, AK, AA, GK, ES, and $A P$ have given final approval of the version to be published, take public responsibility for the content, agree to be accountable for all aspects of the work and vouch for its accuracy and integrity. CP drafted the original manuscript, made substantial contributions to acquisition and interpretation of the data, has given final approval of the version to be published, takes public responsibility for the content, agrees to be accountable for all aspects of the work and vouches for the accuracy and integrity of the data. All authors read and approved the final manuscript.}

\section{Ethics approval and consent to participate}

The study was conducted in accordance with the Declaration of Helsinki and all applicable local requirements. The original study protocol was reviewed and approved by the competent institutional review boards of the participating hospital sites before the enrollment of any patient into the study and the performance of any study-related procedure. The IRBs which approved the conduct of this study were those of the: General Hospital of Athens "Alexandra", Athens, Greece; Prefecture General Hospital for Cancer Treatment "Agioi Anargyri", Athens, Greece (two principal investigators); University General Hospital of loannina, loannina, Greece; General University Hospital of Heraklion, Crete, Greece; Regional Hospital for Cancer Treatment "Agios Savvas", Athens, Greece (two principal investigators); 251 Air Force Hospital, Athens, Greece; "Mitera" Maternity Hospital, Athens, Greece; "IASO" General Hospital, Athens, Greece; General Hospital of Chania "Agios Georgios", Crete, Greece; Piraeus Regional General Hospital for Cancer Treatment "Metaxa", Piraeus, Greece; General Hospital of Patras "Ag. Andreas", Patras, Greece; "Metropolitan" Hospital, Piraeus, Greece; Diagnostic Centre of Athens "Hygeia", Athens, Greece; and "Attikon" University Hospital, Athens, Greece. All subsequent amendments of the study protocol were also approved by the competent institutional review boards. Due to the retrospective chart review design that included the collection of secondary data only and in order to minimize the risk of biasing the clinical outcomes by exclusion from study participation of deceased subjects or patients not able to provide consent, informed consent requirement was not applied.

\section{Consent for publication}

Not applicable.

\section{Competing interests}

AK has nothing to disclose. AA has received honoraria for consultancy in advisory boards from Pfizer, Novartis and Roche. ES has received speaker honoraria from Novartis, BMS, AstraZeneca, Genesis, MSD, Amgen, Merck and Roche. GK has received honoraria for consultancy in advisory boards from Novartis, BMS, AstraZeneca, Genesis, MSD and Roche. AP has received honoraria for consultancy in advisory boards from AstraZeneca, Novartis and Roche and research grants from BMS. CP has received speaker honoraria and honoraria for consultancy in advisory boards from Novartis, AstraZeneca, Genesis, MSD, Amgen, Merck and Roche and research grants from BMS and Roche.

\section{Publisher's Note}

Springer Nature remains neutral with regard to jurisdictional claims in published maps and institutional affiliations.
Acknowledgements

The authors wish to thank Andriana Papaconstantinou and Agoritsa Bismpiroula from the Clinical Research Organization Qualitis Ltd. for medical writing support and statistical analysis of the study data. 


\section{Author details}

'Department of Medical Oncology, University General Hospital of Heraklion Crete Voutes, 711 10, Iraklio, Greece. ${ }^{2}$ 1st Department of Medical Oncology, Agios Savvas Anticancer Hospital, 171 Alexandras Av, 11522 Athens, Greece. ${ }^{3}$ 2nd Department of Medical Oncology, Agios Savvas Anticancer Hospital, 171 Alexandras Av, 11522 Athens, Greece. ${ }^{4} 3$ rd Department of Medical Oncology, Agii Anargiri Cancer Hospital, Kaliftaki 145, 14564 N. Kifissia, Athens, Greece. ${ }^{5}$ Medical Oncology Unit, ATTIKON University Hospital, 1 Rimini St, 12462 Athens, Greece. ${ }^{6}$ Oncology Unit, 2nd Department of Surgery, Aretaieion Hospital, 76 Vas. Sofias Av, 11528 Athens, Greece.

Received: 19 December 2017 Accepted: 10 January 2019

Published online: 18 January 2019

\section{References}

1. Global Burden of Disease Cancer Collaboration, Fitzmaurice C, Allen C, Barber RM, Barregard L, Bhutta ZA, Brenner H, et al. Global, Regional, and National Cancer Incidence, Mortality, Years of Life Lost, Years Lived With Disability, and Disability-Adjusted Life-years for 32 Cancer Groups, 1990 to 2015: A Systematic Analysis for the Global Burden of Disease Study. JAMA Oncol. 2017;3:524-48.

2. Ferlay J, Steliarova-Foucher E, Lortet-Tieulent J, Rosso S, Coebergh JW, Comber $\mathrm{H}$, et al. Cancer incidence and mortality patterns in Europe: estimates for 40 countries in 2012. Eur J Cancer. 2013;49:1374-403.

3. Surveillance Epidemiology and End Results. SEER Stat Fact Sheets: Breast Cancer. SEER Cancer Statistics Review 2006-2012. Available at https://seer. cancer.gov/statfacts/html/breast.html. Accessed December 6, 2016.

4. Lu J, Steeg PS, Price JE, Krishnamurthy S, Mani SA, Reuben J, et al. Breast cancer metastasis: challenges and opportunities. Cancer Res. 2009;69:4951-3.

5. Bonotto M, Gerratana L, Poletto E, Driol P, Giangreco M, Russo S, et al. Measures of outcome in metastatic breast cancer: insights from a real-world scenario. Oncologist. 2014;19:608-15.

6. Kiely BE, Soon YY, Tattersall MH, Stockler MR. How long have I got? Estimating typical, best-case, and worst-case scenarios for patients starting first-line chemotherapy for metastatic breast cancer: a systematic review of recent randomized trials. J Clin Oncol. 2011;29:456.

7. Dafni U, Grimani I, Xyrafas A, Eleftheraki AG, Fountzilas G. Fifteen-year trends in metastatic breast cancer survival in Greece. Breast Cancer Res Treat. 2010; 119:621.

8. Lobbezoo DJ, van Kampen RJ, Voogd AC, Dercksen MW, van den Berkmortel F, Smilde TJ, et al. Prognosis of metastatic breast cancer: are there differences between patients with de novo and recurrent metastatic breast cancer? Br J Cancer. 2015;112:1445-51.

9. National Comprehensive Cancer Network. NCCN Clinical Practice Guidelines in Oncology: Breast Cancer, Version 2.2016. Available at: www.nccn.com. Accessed 8 Dec 2016.

10. Cardoso F, Costa A, Senkus E, Aapro M, André F, Barrios CH, et al. 3rd ESOESMO international consensus guidelines for advanced breast Cancer (ABC 3). Ann Oncol. 2017;28:16-33

11. Dawood S, Broglio K, Ensor J, Hortobagyi GN, Giordano SH. Survival differences among women with de novo stage IV and relapsed breast cancer. Ann Oncol. 2010;21:2169-74.

12. Giuliano M, Trivedi MV, Schiff R. Bidirectional crosstalk between the estrogen receptor and human epidermal growth factor receptor 2 signaling pathways in breast cancer: molecular basis and clinical implications. Breast Care (Basel). 2013;8:256-62.

13. Angus L, Beije N, Jager A, Martens JW, Sleijfer S. ESR1 mutations: moving towards guiding treatment decision-making in metastatic breast cancer patients. Cancer Treat Rev. 2017:52:33-40.

14. Pentheroudakis G, Fountzilas G, Kalofonos HP, Golfinopoulos V, Aravantinos G, Bafaloukos D, et al. Hellenic cooperative oncology group. Palliative chemotherapy in elderly patients with common metastatic malignancies: a Hellenic cooperative oncology group registry analysis of management, outcome and clinical benefit predictors. Crit Rev Oncol Hematol. 2008;66:237-47.

15. Pentheroudakis G, Fountzilas G, Bafaloukos D, Koutsoukou V, Pectasides D Skarlos D, et al. Metastatic breast cancer with liver metastases: a registry analysis of clinicopathologic, management and outcome characteristics of 500 women. Breast Cancer Res Treat. 2006;97:237-44.

16. Kaplan EL, Meier P. Nonparametric estimation from incomplete observations. J Am Stat Assoc. 1958;53:457-81.

17. Colombo PE, Milanezi F, Weigelt B, Reis-Filho JS. Microarrays in the 2010s: the contribution of microarray-based gene expression profiling to breast cancer classification, prognostication and prediction. Breast Cancer Res. 2011;13:212.

18. Parise CA, Caggiano V. Breast cancer survival defined by the ER/PR/HER2 subtypes and a surrogate classification according to tumor grade and immunohistochemical biomarkers. J Cancer Epidemiol. 2014;469251.

19. Howlader N, Altekruse SF, Li Cl, Chen WW, Clarke CA, Ries LA, et al. US incidence of breast cancer subtypes defined by joint hormone receptor and HER2 status. J Natl Cancer Inst. 2014;106 pii: dju055.

20. Geiger S, Cnossen JA, Horster S, DiGioia D, Heinemann V, Stemmler HJ. Long-term follow-up of patients with metastatic breast cancer: results of a retrospective, single-center analysis from 2000 to 2005. Anti-Cancer Drugs. 2011;22:933-9.

21. Swallow E, Zhang J, Thomason D, Tan RD, Kageleiry A, Signorovitch J. Realworld patterns of endocrine therapy for metastatic hormone-receptorpositive (HR+)/human epidermal growth factor receptor-2-negative (HER2-) breast cancer patients in the United States: 2002-2012. Curr Med Res Opin. 2014;30:1537-45.

22. Lobbezoo DJ, van Kampen RJ, Voogd AC, Dercksen MW, van den Berkmortel F, Smilde TJ, et al. In real life, one-quarter of patients with hormone receptor-positive metastatic breast cancer receive chemotherapy as initial palliative therapy: a study of the Southeast Netherlands Breast Cancer Consortium. Ann Oncol. 2016;27:256-62.

23. Sharma P. Biology and Management of Patients With Triple-Negative Breast Cancer. Oncologist. 2016;21:1050-62.

24. Hennigs A, Riedel F, Gondos A, Sinn P, Schirmacher P, Marmé F, et al. Prognosis of breast cancer molecular subtypes in routine clinical care: A large prospective cohort study. BMC Cancer. 2016;16:734.

25. Lee HJ, Park IA, Park SY, Seo AN, Lim B, Chai Y, et al. Two histopathologically different diseases: hormone receptor-positive and hormone receptornegative tumors in HER2-positive breast cancer. Breast Cancer Res Treat. 2014;145:615-23.

26. Wang $Y$, Sun T, Wan D, Sheng L, Li W, Zhu H, et al. Hormone receptor status predicts the clinical outcome of human epidermal growth factor 2positive metastatic breast cancer patients receiving trastuzumab therapy: a multicenter retrospective study. Onco Targets Ther. 2015;8:3337-48.

27. Berman AT, Thukral AD, Hwang WT, Solin LJ, Vapiwala N. Incidence and patterns of distant metastases for patients with early-stage breast cancer after breast conservation treatment. Clin Breast Cancer. 2013;13:88-94.

28. Cheng YC, Ueno NT. Improvement of survival and prospect of cure in patients with metastatic breast cancer. Breast Cancer. 2012;19:191-9.

\section{Ready to submit your research? Choose BMC and benefit from:}

- fast, convenient online submission

- thorough peer review by experienced researchers in your field

- rapid publication on acceptance

- support for research data, including large and complex data types

- gold Open Access which fosters wider collaboration and increased citations

- maximum visibility for your research: over $100 \mathrm{M}$ website views per year

At BMC, research is always in progress.

Learn more biomedcentral.com/submissions 